\title{
A Survey on Super-Resolution Methods for Image Reconstruction
}

\author{
Elham Karimi \\ Department of Electrical \\ Engineering \\ Islamic Azad University, \\ Central Tehran Branch Tehran, \\ Iran
}

\author{
Kaveh Kangarloo \\ Department of Electrical \\ Engineering \\ Islamic Azad University, \\ Central Tehran Branch Tehran, \\ Iran
}

\author{
Shahram javadi \\ Department of Electrical \\ Engineering \\ Islamic Azad University, \\ Central Tehran Branch Tehran, \\ Iran
}

\begin{abstract}
Today, in many applications of Machine vision, image SuperResolution is preferred. Super-Resolution is estimation of a high-resolution image from an image or several low resolution images. Popular techniques in the field of enhancing images can be used to remove noise or blurring. In this paper, an overview of super resolution methods has been presented. Types of resolution methods have been used so far can be divided into three groups as frequency-domain methods, spatial domain methods and techniques can be classified as the wavelet domain. Super-resolution methods in different domains have different characteristics and comparison between these methods is usually done using a special index in one domain. In this paper, we will introduce these indexes and review best techniques used in all three domains.
\end{abstract}

\section{Keywords}

super-resolution; noise elimination; blurring; frequencydomain; spatial domain; wavelet domain.

\section{INTRODUCTION}

Super-resolution is a process in which a high-resolution image from one or more low-resolution image is produced. In the imaging process, it is possible to image detail or high frequency components lost for several reasons, including the low number of camera sensory cells, ambient light from different elements, camera movement and not adjust the camera's focal point. The super-resolution is an attempt to retrieve image details that are lost. In other words, superresolution predicts or interpolates lost data from available evidences and minimizes the image's blurring and aliasing.

Super-resolution methods are used in many machine vision and image processing applications. Today, advances in computers with higher accuracy and processing power, has caused more attention to be software based super-resolution methods. super-resolution is used in monitoring systems such as the identification and recognition of license plates [1], face recognition [2], automatic target recognition [3] and [4], remote sensing [5], medical image processing such as CT and MRI [6] and [7], converting video to different standards (for example, converting NTSC to HDTV), image enhancing [8], processing of satellite images [9], astronomical image processing [10], microscopic image processing [11] and image mosaicking [12].

\section{SUPER-RESOLUTION METHODS}

Super-resolution techniques in terms of the nature are divided into linear and nonlinear methods, methods based on repeatable techniques (recursive) methods and single-frame or multi-frame methods. Super-resolution techniques are also classified according to the used domain which is the spatial domain, frequency-domain and wavelet domain. In this paper, we will investigate methods of super-resolution in these three domains.

\section{FREQUENCY DOMAIN METHODS}

In this section, a review of the various super-resolution methods in the frequency-domain is presented. Superresolution methods in the frequency-domain are related to the common feature of Fourier transforms particularly sampling theory and transport properties. Because these characteristics are completely known, frequency-domain perspectives are very easily understandable. Frequency-domain methods have advantages such as simplicity and low computational complexity, and they can be implemented in parallel. One major flaw in frequency-domain techniques is consideration a model based on the features that each transitional motion in the spatial domain has a dual feature in Fourier domain and it is equal to phase shift. However, any spatial variation in the spatial domain hasn't any duality in the Fourier domain and cannot be introduced in the considered model.

If a scene is represented by $f(x, y)$, the global translations produce $R$ shifted images which can be expressed as $f_{r}(x, y)=f\left(x+\Delta x_{r}, y+\Delta y_{r}\right)$ and $r=1,2, \ldots, R$. The continuous Fourier transform of the scene is represented by $F(u, v)$ and those of translated images are represented by $F_{r}(u, v)$. Using shifting properties of continuous Fourier transform, continuous Fourier transform of the transmitted images can be written as follows.

$$
F_{r}(u, v)=e^{j 2 \pi\left(\Delta x_{r} u, \Delta y_{r} v\right)} F(u, v)
$$

The shifted images are impulse sampled with the sampling period $T_{x}$ and $T_{y}$ to obtain low resolution images $y_{r}[m, n]=f\left(m T_{x}+\Delta x_{r}, n T_{y}+\Delta y_{r}\right]$ with $m=0,1,2, \ldots, M-1$ and $n=0,1,2, \ldots, N-1$. The discrete Fourier transforms (DFTs) of these low-resolution images denotes by $\mathrm{y}_{\mathrm{r}}[k, l]$. The CFTs of the shifted images are 
related with their DFTs by the aliasing property:

$\mathrm{y}_{\mathrm{r}}[k, l]=\alpha \sum_{p=-\infty}^{\infty} \sum_{q=-\infty}^{\infty} F_{r}\left(\frac{k}{M T_{x}}+p f_{s_{x}}, \frac{1}{N T_{y}}+q f_{s_{y}}\right)$

which $f_{s_{x}}=1 / T_{x}$ and $f_{s_{y}}=1 / T_{y}$ are equal to the sampling rate in the axial directions and $\alpha=1 /\left(T_{x} T_{y}\right)$.

Assuming $F(x, y)$ is band-limited, Eqn. (2) can be written as a matrix equation that expresses aliasing.

$$
\mathbf{Y}=\boldsymbol{\Phi} \mathbf{F}
$$

Where $\mathbf{Y}$ is the column vector matrix including the DFTs of $R$ shifted images, $\mathbf{F}$ is the $n m \times 1$ column vector containing the samples of the unknown CFT coefficients of original image, $\boldsymbol{\Phi}$ is a matrix relating $\mathbf{Y}$ and $\mathbf{F}$. Eqn. (3) defines a set of linear equations from which we intend to solve $\mathbf{F}$ and then use the inverse DFT to obtain the reconstructed image. In the super-resolution, discrete Fourier coefficients of $R$ observed images must be obtained and then $\boldsymbol{\Phi}$ must be estimated. Finally, using the Eqn. (3) the $\mathbf{F}$ is obtained and with applying inverse DFT, the high-resolution image will be reconstructed. According to the brief description presented above, the different types of frequencydomain methods for image super-resolution are discussed in the next section.

\subsection{Restoration Via Alias Removal}

First spatial image reconstruction using frequency-domain is presented by Tsai and Huang [13]. In this method, as mentioned earlier, $\mathbf{Y}=\boldsymbol{\Phi} \mathbf{F}$ relation is obtained then $\boldsymbol{\Phi}$ is estimated and by using the inverse Fourier transform, the high resolution image is obtained. Although the computationally attractive and simple method is introduced, it has many disadvantages. Assuming ideal sampling ignoring the effect of the imaging sensor and ignoring the effect of noise in the image is the most important of them, because the proposed method only considered the motion transition between images.

In [14] a method based on the model introduced by Tsai and Huang introduced. The Taylor series expansion is used to calculate the transition variables. This method has fewer computations than the Tsai and Huang frequency-domain method. In [15] the main approach problem expressed and a method based on frequency-domain was introduced in [16] is also extended. In this way blurring impact and noise is also considered. By using the least squares sense criterion and similar to Tsai and Huang method, a high resolution is reconstructed.

\subsection{Recursive Least Squares (RLS)}

In this approach $\mathbf{Y}=\boldsymbol{\Phi} \mathbf{F}$ is solved based on the recursive least squares error equations and using of a recursive method. In these methods, blurring and noise are considered. A solution based on recursive least squares method of determining the $\mathbf{F}$ with regard to noise, is minimizing Eqn. (4) [17-19].

$$
\|\mathbf{\Phi F}-\mathbf{Y}\|^{2}+\gamma(\Phi)
$$

where $\gamma($.$) is regularization functional. In [18, 19], the$ relation is considered as Eqn. (5), where $\mathbf{c}$ is an approximate solution to the equation (not yet known).

$$
\gamma(\mathbf{F})=\lambda\|\mathbf{F}-\mathbf{c}\|^{2}
$$

Replacement regularization function in equation (4) and minimizing it the Eqn. (6) is obtained.

$$
\hat{F}=\left(\boldsymbol{\Phi}^{T} \boldsymbol{\Phi}+\lambda \mathbf{I}\right)^{-1}\left(\boldsymbol{\Phi}^{T} Y+\lambda \mathbf{c}\right)
$$

This equation is solved with a recursive relationship and without inversing matrixes. Initial value of the variable $\mathbf{c}$ is set to zero, then at each step, put $\mathbf{c}$ amount equal to the result of the previous step. One disadvantage of this method is the use of an estimate for the unknown $\mathbf{c}$ that does not guarantees the convergence and the stability of the recursive solution.

\subsection{Recursive Total Least Squares (RTLS)}

In [20] to provide a robust method for solving $\mathbf{Y}=\mathbf{\Phi F}$ the Recursive Total Least Squares method was used, where in addition to the noise, an error in $\boldsymbol{\Phi}$ is also considered. The model used in this method is presented in Eqn. (7).

$$
\mathbf{Y}=[\mathbf{\Phi}+\mathbf{E}] \mathbf{F}+\mathbf{N}
$$

where $\mathbf{E}$ is the produced error in the $\boldsymbol{\Phi}$ due to errors in the motion estimation and $\mathrm{N}$ denotes the noise that is proportional to the application. In this method $\mathbf{F}$ is obtained based on the recursive method, Eqn. (7) and minimizing equation. $\|[\mathbf{N}: \mathbf{E}]\| .\|\cdot\|$ is the Frobenius norm and defined as square root of the sum of the squares of the absolute values of all elements of the matrix. In [20] the Recursive Total Least Squares is used for image reconstruction and [21] theoretically analyzed the performance of this method.

\subsection{Multichannel Sampling Theorem Methods}

In [22] a method based on multi-channel sampling theorem is introduced. However, this method is implemented in the spatial domain, but fundamentally, this method is a method in the frequency-domain, because transmission characteristics of the Fourier transform of the original image is used to model the translation. In this method, the band-limited function is passed through the number of "mutually independent" linear channels, the outputs of which are under-sampled at the rate below the Nyquist rate to produce discrete signals by a number equal to the number of channels. Using the theory of multichannel sampling the original signal can be fully reconstructed from these discrete signals. In this method, discrete signals that are actually sampled signals from the original signal are passed through $R$ linear filters and then summing the resulting outputs and interpolating produced signals, the original signal can be reconstructed. Consider a function $f(x)$ which is band-limited to $-\sigma<\omega<\sigma$ and is passed through $R$ linear channels, the outputs of which are sampled at the rate $2 \sigma / R$ ((under-sampled at $1 / R$ of the Nyquist rate) to produce $R$ discrete signals $y_{r}(m T)$, $T=R / 2 \sigma, \quad m \in Z, \quad r \in\{1,2, \ldots R\} . \quad$ In Multichannel Sampling Theorem, produced $y_{r}(m T)$ signals are passed throw $R$ linear filters with impulse functions $h_{r}($.$) and the$ total output of these filters is obtained according to Eqn. (8).

$$
\hat{f}(x)=\sum_{r=1}^{R} \hat{f}_{r}(x)=\sum_{r=1}^{R} \sum_{m=-\infty}^{\infty} y_{r}(m T) \cdot h_{r}(x-m T)
$$


where $\hat{f}(x)$ is a sampled version of $f(x)$ meeting the Nyquist criterion and which may be interpolated to recover $f(x)$ exactly.

Further details of the methods in the frequency-domain are given in [23].

\section{Spatial Domain Super-Resolution Methods}

Effects of optical blur and system degradations, motion blurring, spatial variation of sensor point spread function, effects of non-ideal sampling, compression artifacts and more can be modeled in spatial domain super-resolution to image reconstruction.

Suppose that the original image is estimated from several low resolution images. If the low-resolution images be shown by $\mathbf{y}_{r}, r \in\{1,2, . ., R\}, \mathbf{y}_{r}$ and $\mathbf{F}$ (original image) are related as $\mathbf{y}_{r}=\mathbf{H}_{r} \mathbf{F}$. The matrix $\mathbf{H}_{r}$ which must be estimated, incorporates motion compensation, degradation effects and subsampling. The observation equation may be generalized to

$$
\mathbf{Y}=\mathbf{H F}+\mathbf{N} \quad \text { where } \quad \mathbf{Y}=\left[\mathbf{y}_{1}^{T} . . \mathbf{y}_{R}^{T}\right]^{T} \quad \text { and }
$$$$
\mathbf{H}=\left[\mathbf{H}_{1}^{T} . . \mathbf{H}_{R}^{T}\right]^{T} \text { with } \mathbf{N} \text { representing observation noise. }
$$

\subsection{Iterated Back Projection (IBP)}

In the paper [24] the application of this method has been shown in image reconstruction. With estimated high resolution image $\hat{\mathbf{F}}$ and the imaging model $\mathbf{H}$, it is possible to finding the corresponding low-resolution images $\hat{\mathbf{Y}}$ as $\hat{\mathbf{Y}}=\mathbf{H} \hat{\mathbf{F}}$. Iterated backprojection (IBP) procedures update the estimate of the SR reconstruction by backprojecting the error between the images $\hat{\mathbf{Y}}^{(j)}$ obtained in the $\mathrm{j}$-th iteration from model $\mathbf{H}$, and the observed LR images $\mathbf{Y}$. Indeed in each iteration, based on the error values are added to the estimated image $(\hat{\mathbf{F}})$ pixels until the error reaches the desired value. Eqn. (9) is used for this method.

$$
\begin{aligned}
& \hat{\mathbf{F}}^{(j+1)}=\hat{\mathbf{F}}^{(j)}+\mathbf{H}^{B P}\left(\mathbf{Y}-\hat{\mathbf{Y}}^{(j)}\right) \\
& =\hat{\mathbf{F}}^{(j)}+\mathbf{H}^{B P}\left(\mathbf{Y}-\mathbf{H} \hat{\mathbf{F}}^{(j)}\right)
\end{aligned}
$$

Typically $\mathbf{H}^{B P}$ approximates $\mathbf{H}^{-1}$. Eqn. (9) is repeated until some error criteria that are determined based on $\hat{\mathbf{Y}}^{(j)}$ and $\mathbf{Y}$ is minimized. Unfortunately the SR reconstruction is not unique since SR is an ill-posed inverse problem.

\subsection{Statistical Approaches}

In this method, the HR image and motions among lowresolution inputs are considered as a stochastic variable. Suppose that in the $M(v, h)$ (Matrix in which the effects of motion and blurring in the LR image are considered and is known as the degradation matrix.) $v$, and $h$ denote the motion vector and blurring kernel respectively. Super resolution in the Bayesian framework is expressed as follows.

$$
\mathbf{F}=\arg \max _{\mathbf{F}} \int_{v, h} P_{r}(\mathbf{Y} \mid \mathbf{F}, M(v, h)) P_{r}(\mathbf{F}) P_{r}(M(v, h)) d v(10)
$$

Here $P_{r}(\mathbf{Y} \mid \mathbf{F}, M(v, h))$ is the data likelihood, $P_{r}(\mathbf{F})$ is the prior term on the desired high-resolution image, $P_{r}(M(v, h))$ is a prior term on the motion estimation and $\arg \max _{x} f(x):=\{x \mid \forall y: f(y) \leq f(x)\}$. Above equation is complicated and difficult to evaluate due to the integration over motion estimates. If $M(v, h)$ is given or estimated beforehand (denote as $M$ ), Eqn. 10 can be simplified as

$$
\begin{aligned}
& \mathbf{F}=\arg \max _{\mathbf{F}} P_{r}(\mathbf{Y} \mid \mathbf{F}, M) P_{r}(\mathbf{F}) \\
& =\arg \min _{\mathbf{F}}\left\{\|\mathbf{Y}-\mathbf{M F}\|^{2}+\lambda A(\mathbf{F})\right\}
\end{aligned}
$$

where $\arg \min _{x} f(x):=\{x \mid \forall y: f(x) \leq f(y)\}, A(\mathbf{F})$ is a non-negative potential function and $\lambda$ absorbs the variance of the noise. Eqn.11 is the popular Maximum a Posteriori (MAP) formulation for SR, where $M$ is assumed to be known. The following is an overview of the types of statistical approaches.

\subsubsection{Maximum Likelihood (ML)}

If we assume uniform prior over $\mathbf{F}$, Eqn. 11 reduces to the simplest maximum likelihood (ML) estimator (motion estimation is assumed as a prior). In this method by minimizing $p_{r}(\mathbf{Y} \mid \mathbf{F})$, the desired image is estimated as follows.

$$
\hat{\mathbf{F}}=\arg \min _{\mathbf{F}}\left\{\|\mathbf{Y}-\mathbf{M F}\|^{2}\right\}
$$

Several methods have been proposed to solve this equation. Derivative of this equation with respect to $\mathbf{F}$ and putting it equal to zero, the following recursive equation is obtained that has several solutions.

$$
\hat{\mathbf{F}}=\left(M^{T} M\right)^{-1} M^{T} Y
$$

Irani and Peleg have developed a simple but very popular method based on an error back-projection scheme to solve this equation [25.26]. In this algorithm iteratively the current estimate is updated by adding back the warped simulation error convolved with a back-projection function. In [27] proposed a maximum likelihood SR image estimation algorithm to estimate the subpixel shifts, noise of the image, and the HR image simultaneously. The proposed maximum likelihood estimation is treated by the ExpectationMaximization (EM) algorithm. The maximum likelihood is very sensitive to noise and registration errors.

\subsubsection{Maximum A Posteriori}

Several methods based on the maximum a posteriori in various works $[9,14,28]$ have been considered where the techniques vary in the observation model assumptions and the prior term $p_{r}(\mathbf{F})$ for the desired solution. Gaussian Markov Random Field (GMRF) [29], Huber MRF (HMRF) [28], Total Variation (TV) [30] are different kinds of priors have been proposed in the literature. The following equation is used in the Markov random field approach.

$$
\mathbf{A}(\mathbf{F})=\mathbf{F}^{\mathbf{T}} \mathbf{Q} \mathbf{F}
$$

In this equation $\mathbf{Q}$ is a symmetric positive matrix and usually defined as $\Gamma^{T} \Gamma$ and $\Gamma$ is similar to first or second derivative operator that acts on the image F. In this case, Maximum a Posteriori is expressed as follows. 


$$
\|\Gamma \mathbf{F}\|^{2} \propto \log P(\mathbf{F})
$$

This relationship is known as Tikhonov regularization [31]. Usually $\Gamma$ is referred to as Tikhonov matrix.

\subsubsection{Joint MAP Restoration}

Resolution can be classified into two problems that are the low-resolution images registration and estimation of the highresolution image. In many of the techniques mentioned in the previous sections, two processes are considered as two distinct processes, but in the Joint MAP Restoration, motion estimation and image restoration benefits each other and combining together. In this method, Eqn. (10) is extended to considering motion estimation.

$$
\begin{aligned}
& \{\mathbf{F}, v, h\}=\underset{\mathbf{F}, v, h}{\arg \min }-\log \left[P_{r}(\mathbf{Y} \mid \mathbf{F}, M(v, h))\right] \\
& -\log \left[P_{r}(\mathbf{F})\right]-\log \left[P_{r}(M(v, h))\right]
\end{aligned}
$$

In [27] three-stage image $\mathrm{SR}$ is divided into three stages namely registration, restoration and image interpolation. Registration and restoration estimated simultaneously by using maximum likelihood (ML) and the expectation maximization (EM). Later, the same authors included interpolation into the framework and estimated all of the unknowns using EM in [32]. In [33] a Joint MAP Restoration based method is introduced that simultaneously estimates HR image restoration and transmission parameters using an optimization process.

\subsubsection{Bayesian Methods}

In [23] the Bayes theorem was introduced as synonymous and exactly the same to popular Maximum a Posteriori. However, this method is similar to the Maximum a Posteriori and joint MAP methods, can simultaneously obtain the registration parameters and estimate the high-resolution images, but has some differences. Joint MAP estimation of motion parameters and HR image may face the problem of overfitting [34]. . In [34] a method based on Bayes theory is presented in which the integration of high-resolution image and the marginal is used to estimate motion parameters. Determine the parameters with higher precision and higher quality image reconstruction are the listed advantages of this method. To make the integration tractable, image priors or registration parameters have to take simple parametric forms, limiting these models in dealing with more complex cases. Computing in realistic applications may also cause problems.

\subsection{Example-Based Approaches}

In the previous methods, the numbers of frames are used for image reconstruction and super-resolution that these frames contain complementary spatial information. But in many cases, there is only a single low resolution image and use of these methods for image reconstruction is not effective. Today, new example based methods are introduced in which to overcome the shortcomings of the measurement and imaging, many examples are used. Unlike previous approaches that use a rule to specify parameters from the whole image, these methods estimate the desired image by sampling from other images in a local way [35], [36]. In [37] an example based method is introduced in which the examples were directly used for image reconstruction. In this method, two sets of training patches sampled from the high-resolution and low-resolution images are used for image superresolution.

\subsection{Set Theoretic Reconstruction Methods}

\subsubsection{Projection On to Convex Sets (POCS)}

Techniques based on the projection on to convex sets are the eminent group of Super-resolution methods. Projection onto convex sets using an iterative procedure and for any given point within the vector space, a point identified, which satisfies all the convex constraint sets. These methods have many advantages, including simplicity, flexible spatial domain observation model (For example, the complexity of the motion and observation model does not significantly affect the POCS solution procedure), powerful inclusion of a priori information and any kinds of constraints. However, heavy computation and slow convergence are the two renowned defects of the POCS methods. In this approach, the solution is not unique and depending on the initial guess. In [15] and [38] POCS based solutions are proposed to super-resolution image reconstruction problems.

\subsubsection{Bounding Ellipsoid Method}

In [39] a variant of the POCS based formulation has been investigated. In this method, an ellipsoid is used to bound the constraint sets. The centroid of this ellipsoid is taken as the super-resolution estimate. Since direct computation of this point is infeasible, an iterative procedure is used.

\subsection{Optimal and Adaptive Filtering}

The optimal adaptive filters have been used in several superresolution image reconstruction studies. One of the main disadvantages of these methods is their limitations in considering the priori constraints as compared with POCS or Bayesian methods. In [40] the adaptive filter is used for image super resolution. Non-linear a-priori constraints are not included in these methods.

\subsection{Neural Network Based Methods}

In [41] a multi-layer perceptron neural network with probabilistic neural network (MLP-PNN) is used for image super-resolution. Hybrid MLP-PNN neural architecture is shown in Fig.1. This method has two steps. In the first step, scattered-point interpolation is carried out on projected sequence data using neural network architecture. Neural network learning procedure is performed with examples optimum distance-to-weight interpolation functions at several input sequence noise levels. Restoring the combined degradations due to low-resolution optics and detector blurs, as well as residual degradations due to the interpolation procedure on the interpolated image is performed in the second step. In this approach a near real-time implementation can be realized due to the simplicity of the method. The use of optimum coefficients for both steps to the process enables to obtain results of quality equal or better than classical MAP super-resolution methods [41].

\subsection{Interpolation Based Methods}

Interpolation based methods use several low resolution images and with a behavior like a reverse downsampller produce a higher resolution image. These techniques attempt to reconstruct a HR image from an irregular set of LR samples and usually consist of the following three stages: (i) the registration stage, (ii) the interpolation stage for producing a higher-resolution image, and (iii) the deblurring stage for enhancing the reconstructed high-resolution image produced in the step (ii). 


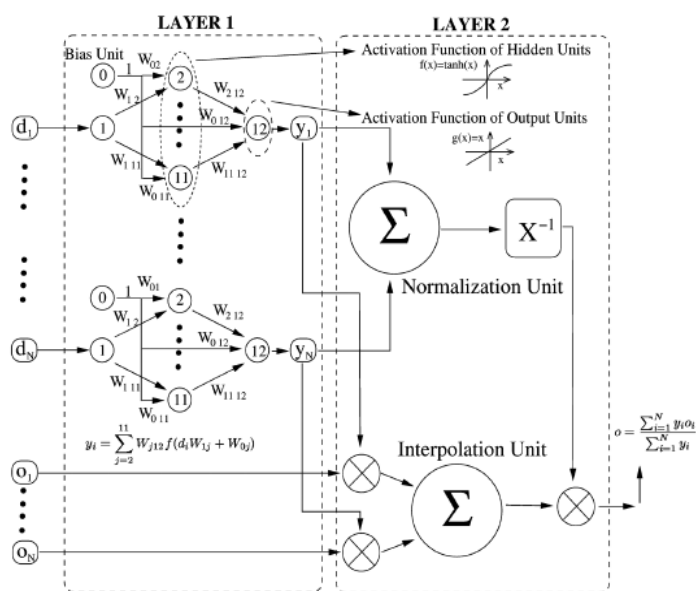

Fig 1. Block diagram of neural network that is used in [41].

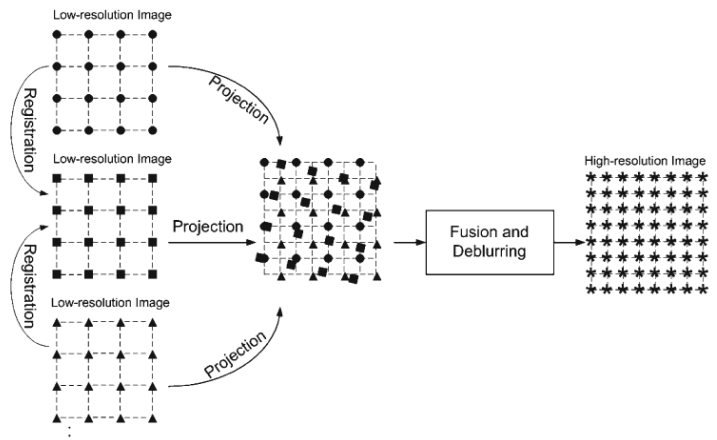

Fig 2. Image registration before super-resolution [43].

Note that the single image interpolation algorithm cannot handle the SR problem well, since it is inherently limited by the amount of data available in the image and cannot produce those high-frequency components that were lost during the image acquisition process. Different types of interpolation methods for increasing image resolution used in literature, in this section some of the most important of them is outlined.

\subsubsection{Interpolation of NonUniformly Spaced Samples}

Registering a set of LR images using motion compensation produces in a single, dense composite image of nonuniformly spaced samples as shown in Fig. 2. High resolution image is produced with using techniques for reconstruction from nonuniformly spaced samples. Iterative reconstruction techniques, based on the Landweber iteration, have been applied [42]. These are very simple interpolation methods and there are incapable of reconstructing significantly more frequency content than is present in a single LR frame.

\subsubsection{Nearest Neighbor Interpolation}

Nearest neighbor interpolation is the simplest method for image interpolation. For each point on the HR grid, the closest known LR pixel is selected and the value of that pixel is simply used as the value at the grid point. This method, therefore, implicitly assumes a piece-wise constant model for the image. It is the fastest of all interpolation methods as it considers only a single pixel - that closest to the grid point being interpolated [44].

Near optimal non-uniform nearest neighbor interpolation and an efficient interpolation scheme based on weighted nearest neighbors, followed by wiener filtering for deblurring are presented in [44] and [45] respectively.

\subsubsection{Other Interpolation Methods}

Other interpolation methods include: Inverse distance weighting, Spline interpolation, bicubic Interpolation and bilinear interpolation. Inverse distance weighting and Spline interpolation methods are described in [46]. An advanced bilinear image interpolation is proposed in [47] and bicubic and some other interpolation methods are explained in [16].

\subsection{Hybrid methods}

These methods typically used to reduce the disadvantages of each method and also enhance them. In this way the methods mentioned above are used in combination. Combining techniques such as ML, MAP and POCS are some examples of these methods $[9,40,48]$. In the papers presented in recent years, the combination of spatial domain methods, neural network based methods and other methods with wavelet based methods are used.

\section{WAVELET DOMAIN METHODS}

Wavelet domain methods can include Regularity Preserving Interpolation, New Edge Direction Interpolation (NEDI), Wavelet domain Zero Padding and Cycle Spinning (WZPCS), Dual Tree Complex Wavelet Transform (DT-CWT), Interpolation of wavelet domain high frequency subband and the spatial domain, Discrete Wavelet Transform (DWT) and Stationary Wavelet Transform (SWT)). These methods are described and compared with each other in [49]. Other methods based on wavelet domain individually or in combination with other methods are proposed in literature. For example, in [50] DASR and bicubic interpolation method are used in parallel.

\section{OTHER SUPER-RESOLUTION AND DEBLURRING METHODS}

Other image super-resolution and deblurring methods are introduced in the literature. Nonnegative and Support Constraints Recursive Inverse Filtering (NAS_RIF), Iterative Blind Deconvolution (IDB) and Richardson Lucy are explained in [51]. In [52] some other methods like Poisson MAP, Wiener filter, Van-Cittert, Richardson Lucy, Scale space and some of the methods previously mentioned, such as Landweber, Tichonove Miller, Total variation and Expectation-Maximization Maximum Likelihood Estimator (EM-MLE) are discussed. Readers can get more details about these methods refer to the references cited.

\section{INDEXES USED TO COMPARE SR METHODS}

Because the various super-resolution methods are too high, it is not possible to compare these methods with each other with unique index and same condition. Usually the methods are compared to each domain or each group. For example, in [49] some wavelet domain methods and some interpolation methods are investigated and compared with Peak signal-tonoise ratio (PNSR) index. Root Mean Square Error (RMSR) and entropy are other indexes that used in literature. However, due to the great variety of methods, the comparison with these indexes is avoided. Usually the references that have been introduced in each category, two or more methods have been compared using these or other indexes. 


\section{CONCLUSION}

In the literature, several image super-resolution methods have been introduced, which shows the importance of these methods in many applications. However, many methods have been introduced for image super-resolution still there are ongoing researches in this field and every day new articles are published on this subject which is indicates of its importance. So in this paper image super-resolution methods are classified and were introduced to an overview of them has been provided.

In this paper Super-resolution methods were examined for the frequency, spatial and wavelet domains and various methods have been introduced in each domain were studied and the characteristics of these methods were identified. Some type of indexes that are used to compare methods were introduced, but because comparison usually occurs between the methods in each domain or specific category was avoided.

\section{REFERENCES}

[1] Sun Hao; Luo Lin; Zhou Weiping; Luo Limin, "Location and Super-Resolution Enhancement of License Plates Based on Video Sequences," Information Science and Engineering (ICISE), 2009 1st International Conference on , vol., no., pp.1319,1322, 26-28 Dec. 2009.

[2] Hong Yu,; Ma Xiang,; Huang Hua,; Qi Chun,, "Face image super-resolution through POCS and residue compensation," Visual Information Engineering, 2008. VIE 2008. 5th International Conference on , vol., no., pp.494,497, July 29 2008-Aug. 12008.

[3] Marco Crisani, Dong Seon Cheng, Vittorio Murino, and Donato Pannullo. "Distilling information with superresolution for video surveillance".In Proceedings of the ACM 2nd International Workshop on Video Surveillance and Sensor Networks, pages 2-11, 2004.

[4] Frank Lin, Clinton B. Fookes, Vinod Chandran, and Sridha Sridharan. "Investigation into optical flow superresolution for surveillance applications".In The Austrilian Pattern Recognition Society Worshop on Digital Image Computing, 2005.

[5] Feng Li; Xiuping Jia; Fraser, D., "Universal HMT based super resolution for remote sensing images," Image Processing, 2008. ICIP 2008. 15th IEEE International Conference on , vol., no., pp.333,336, 12-15 Oct. 2008.

[6] J. Maintz and M. Viergever. A survey of medical image registration. Medical Image Analysis, Vol. 2, No. 1. , pp. 1-36, March 1998

[7] Roohi, S.; Zamani, J.; Noorhosseini, M.; Rahmati, M., "Super-resolution MRI images using Compressive Sensing," Electrical Engineering (ICEE), 2012 20th Iranian Conference on , vol., no., pp.1618,1622, 15-17 May 2012.

[8] Schultz, R.R.; Stevenson, R.L., "Extraction of highresolution frames from video sequences," Image Processing, IEEE Transactions on , vol.5, no.6, pp.996,1011, Jun 1996.

[9] Yun Zhang; Mishra, R.K., "A review and comparison of commercially available pan-sharpening techniques for high resolution satellite image fusion," Geoscience and Remote Sensing Symposium (IGARSS), 2012 IEEE International , vol., no., pp.182,185, 22-27 July 2012.

[10] Sheppard, D.G.; Hunt, B.R.; Marcellin, M.W., "Iterative multiframe super-resolution algorithms for atmospheric turbulence-degraded imagery," Acoustics, Speech and
Signal Processing, 1998. Proceedings of the 1998 IEEE International Conference on , vol.5, no., pp.2857,2860 vol.5, 12-15 May 1998.

[11] Suvrajit Maji, "Generative Models for Super-Resolution Single Molecule Microscopy Images of Biological Structures", Lane Center for Computational Biology, School of Computer Science, Carnegie Mellon University, Pittsburgh PA 15213, CMU-CB-12-104, August 2012.

[12] Kunter, M.; Jangheon Kim; Sikora, T., "Super-resolution Mosaicing using Embedded Hybrid Recursive Folowbased Segmentation," Information, Communications and Signal Processing, 2005 Fifth International Conference on , vol., no., pp.1297,1301, 0-0 0.

[13] R. Tsai and T. Huang, "Multiframe image restoration and registration". In R. Y. Tsai and T. S. Huang, editors, Advances in Computer Vision and Image Processing, volume 1, pages 317\{339, JAI Press Inc. (1984).

[14] Kaltenbacher, E.; Hardie, R.C., "High resolution infrared image reconstruction using multiple, low resolution, aliased frames," Aerospace and Electronics Conference, 1996. NAECON 1996., Proceedings of the IEEE 1996 National , vol.2, no., pp.702,709 vol.2, 20-23 May 1996.

[15] Tekalp, A.M.; Ozkan, M.K.; Sezan, M.I., "Highresolution image reconstruction from lower-resolution image sequences and space-varying image restoration," Acoustics, Speech, and Signal Processing, 1992. ICASSP-92., 1992 IEEE International Conference on , vol.3, no., pp.169,172 vol.3, 23-26 Mar 1992.

[16] R. Sudheer Babu, Dr.K.E.Sreenivasa Murthy, "A Survey on the Methods of Super-Resolution Image Reconstruction", International Journal of Computer Applications (0975 - 8887), Volume 15- No.2, February 2011.

[17] Kim, S. P.; Bose, N.K.; Valenzuela, H.M., "Recursive reconstruction of high resolution image from noisy undersampled multiframes," Acoustics, Speech and Signal Processing, IEEE Transactions on , vol.38, no.6, pp.1013,1027, Jun 1990

[18] Kim, S. P.; Su, W.-Y., "Recursive high-resolution reconstruction of blurred multiframe images," Acoustics, Speech, and Signal Processing, 1991. ICASSP-91., 1991 International Conference on , vol., no., pp.2977,2980 vol.4, 14-17 Apr 1991

[19] Kim, S.P.; Su, W.-Y., "Recursive high-resolution reconstruction of blurred multiframe images," Image Processing, IEEE Transactions on , vol.2, no.4, pp.534,539, Oct 1993

[20] Bose, N.K.; Kim, H. C.; Valenzuela, H.M., "Recursive implementation of total least squares algorithm for image reconstruction from noisy, undersampled multiframes," Acoustics, Speech, and Signal Processing, 1993. ICASSP-93., 1993 IEEE International Conference on , vol.5, no., pp.269,272 vol.5, 27-30 April 1993.

[21] Bose, N.K.; Kim, H. C.; Zhou, B., "Performance analysis of the TLS algorithm for image reconstruction from a sequence of undersampled noisy and blurred frames," Image Processing, 1994. Proceedings. ICIP-94., IEEE International Conference, vol.3, no., pp.571,574 vol.3, 13-16 Nov 1994 
[22] H. Ur and D. Gross, "Improved resolution from subpixel shifted pictures". CVGIP: Graphical Models and Image Processing, 54: 181\{186 (March 1992).

[23] Sean Borman, Robert Stevenson, "Spatial Resolution Enhancement of Low-Resolution Image Sequences, A Comprehensive Review with Directions for Future Research," Laboratory for Image and Signal Analysis (LISA), University of Notre Dame, NotrebDame, IN 46556, July 8, 1998.

[24] M. Irani and S. Peleg, " Motion analysis for image enhancement:Resolution, occlusion and transparency," Journal of Visual Communications and Image Representation, vol.4, issue4, pages 324-335, December1993.

[25] M. Irani, S. Peleg., "Super resolution from image sequences," Pattern Recognition, 1990. Proceedings., 10th International Conference on, vol.ii, no., pp.115,120 vol.2, 16-21 Jun 1990.

[26] M. Irani and S. Peleg," Improving resolution by image registration".CVGIP: Graphical Models and Imaging Processing, vol53, issue3,pages 231-239, May 1991.

[27] Tom, B.C.; Katsaggelos, A.K.; Galatsanos, N.P., "Reconstruction of a high resolution image from registration and restoration of low resolution images," Image Processing, 1994. Proceedings. ICIP-94., IEEE International Conference, vol.3, no., pp.553,557 vol.3, 13-16 Nov 1994.

[28] Capel, D.; Zisserman, A., "Computer vision applied to super resolution," Signal Processing Magazine, IEEE , vol.20, no.3, pp.75,86, May 2003.

[29] K. M. Hanson and G.W. Wecksung,"Bayesian approach to limited-angle reconstruction in computed tomography," Journal of Optical Society of America, JOSA, Vol. 73, Issue 11, pp. 1501-1509 (1983).

[30] L. Rudin, S. Osher, and E. Fatemi. "Nonlinear total variation based noiseremoval algorithms," Journal Physica D, Nonlinear Phenomena, Volume 60, Issue 1-4, Nov. 1, Pages 259-268, 1992.

[31] N. Nguyen, P. Milanfar, and G. H. Golub. "A computationally efficient image superresolution algorithm," IEEE Transactions on Image Processing, 10(5):573-583, 2001..

[32] Tom, B.C.; Katsaggelos, A.K., "Reconstruction of a high-resolution image by simultaneous registration, restoration, and interpolation of low-resolution images," Image Processing, 1995. Proceedings., International Conference on , vol.2, no., pp.539,542 vol.2, 23-26 Oct 1995.

[33] Hardie, R.C.; Barnard, K.J.; Armstrong, E.E., "Joint MAP registration and high-resolution image estimation using a sequence of undersampled images," Image Processing, IEEE Transactions on , vol.6, no.12, pp.1621,1633, Dec 1997.

[34] Michael E. Tipping and Christopher M. Bishop. "Bayesian image superresolution," In Proceedings of Advances in Neural Information ProceedingSystems, pages 1279-1286, 2003.

[35] Efros, A.A.; Leung, T.K., "Texture synthesis by nonparametric sampling," Computer Vision, 1999. The Proceedings of the Seventh IEEE International Conference on , vol.2, no., pp.1033,1038, 1999.
[36] Hertzmann, C. E. Jacobs, N. Oliver, B. Curless, and D. H. Salesin. "Image analogies". In Proceedings of the 28th annual conference Computer Graphics and Interactive Techniques, pages 327-340, 2001.

[37] Freeman, W.T.; Jones, T.R.; Pasztor, E.C., "Examplebased super-resolution," Computer Graphics and Applications, IEEE , vol.22, no.2, pp.56,65, Mar/Apr 2002.

[38] Patti, A.J.; Sezan, M.I.; Tekalp, A.M., "High-resolution image reconstruction from a low-resolution image sequence in the presence of time-varying motion blur," Image Processing, 1994. Proceedings. ICIP-94., IEEE International Conference, vol.1, no., pp.343,347 vol.1, 13-16 Nov 1994

[39] Elad, M.; Feuer, A., "Restoration of a single superresolution image from several blurred, noisy, and undersampled measured images," Image Processing, IEEE Transactions on , vol.6, no.12, pp.1646,1658, Dec 1997.

[40] Patti, A.J.; Tekalp, A.M.; Sezan, M.I., "A new motioncompensated reduced-order model Kalman filter for space-varying restoration of progressive and interlaced video," Image Processing, IEEE Transactions on , vol.7, no.4, pp.543,554, Apr 1998

[41] G. Crist'obala, E. Gila, F. 'Sroubekb, J. Flusserb, C. Miravetc, F. B. Rodr'1guezc. "Superresolution imaging: a survey of current techniques," Proc. SPIE 7074, Advanced Signal Processing Algorithms, Architectures, and Implementations XVIII, 70740C September 03, 2008.

[42] T. Komatsu, T.Igarashi, K. Aizawa, and T. Saito. "Very high resolution imaging scheme with multiple different aperture cameras". Signal Processing Image Communication,vol 5,issue5-6,pages 511- 526, Dec 1993.

[43] Jing Tian, Kai-Kuang Ma, "A survey on super-resolution imaging," Signal, Image and Video Processing In Signal, Image and Video Processing, Vol. 5, No. 3, pp. 329-342 3 February 2011.

[44] A. Gilman and D.G. Bailey, "Near optimal non-uniform interpolation for image super-resolution from multiple images", in Image and Vision Computing New Zealand (IVCNZ'06), Great Barrier Island, NZ, pp 31-36 (27-29 November, 2006).

[45] M. S. Alam, J. G. Bognar, R. C. Hardie, and B. J. Yasuda. Infrared image registration and high-resolution reconstruction using multiple translationally shifted aliased video frames. IEEE Transactions on Instrumentation and Measurement, 49(5):915-923, 2000.

[46] Anders Ohman, "Methods and algorithms for image fusion and super resoloution ," Master of science thesis,department signals and systems,chalmers university of technology,2009.

[47] Heechang Kim; Sangjun Park; Jin Wang; Yonghoon Kim; Jechang Jeong, "Advanced Bilinear Image Interpolation Based on Edge Features," Advances in Multimedia, 2009. MMEDIA '09. First International Conference on , vol., no., pp.33,36, 20-25 July 2009.

[48] Keys, R., "Cubic convolution interpolation for digital image processing," Acoustics, Speech and Signal Processing, IEEE Transactions on , vol.29, no.6, pp.1153,1160, Dec 1981. 
[49] Bagawade Ramdas P., Bhagawat Keshav S., Patil Pradeep M., "Wavelet transform techniques for image resolution enhancement: a study", International Journal of Emerging Technology and Advanced Engineering, Volume 2, Issue 4, April 2012.

[50] Hasan Demirel and Gholamreza Anbarjafari, "Image Super Resolution Based on Interpolation of Wavelet Domain High Frequency Subbands and the Spatial
Domain Input Image,", ETRI Journal, Volume 32, Number 3, June 2010.

[51] H.Y.LIU, Y.S.ZHANG, Song JI, "Sudy on the methods of super-resolution image reconstruction",The International Archives of the Photogrammetry, Remote Sensing and Spatial Information Sciences. Vol. XXXVII. Part B2. Beijing 2008.

[52] S.H.M. Allon, M.G. Debertran, B.T.H.M. Sleutjes, "Fast Deblurring Algorithms," pp. 1-25, 2004. 